\title{
Electron Tomography for Nanoscale Materials Science
}

\author{
P.A.Midgley*, T.J.V. Yates*, I. Arslan*, J. Tong* and J.M. Thomas****
}

*Department of Materials Science and Metallurgy, University of Cambridge, Pembroke Street, Cambridge, CB2 3QZ, UK.

**Davy Faraday Research Laboratory, Royal Institution of Great Britain, 21 Albemarle Street, London, W1X 4BS,UK.

The need for three-dimensional (3D) analysis across all length scales in both physical and life sciences is becoming increasingly common. Microscopic techniques using light, X-rays, neutrons and electrons have all been adapted to allow three-dimensional reconstructions of objects using tomographic methods. The advent of nanotechnology and the possibility of building complex 3D nanoscale devices demands tomographic methods with high spatial resolution. Indeed one can speculate that in 50 years nanoscale devices made using the 'top-down' or 'bottom-up' approach may rival the complexity of some biological systems, see Figure 1. Electron tomography of such devices can provide the required resolution in three dimensions using imaging techniques suited to materials science, such as scanning transmission electron microscopy high angle annular dark field (STEM-HAADF) imaging [1,2] or energy filtered transmission electron microscopy (EFTEM) [3,4].

Tomography is a two-stage process based on the acquisition of a tilt series followed by a threedimensional reconstruction. The acquisition process is now largely an automated process for STEM and TEM-based tomography and it is quite common to record a series of images every 1 or 2 degrees from +70 to -70 degrees. Such angular sampling and high tilt range leads to high fidelity reconstructions, computed using either standard weighted back-projection or iterative techniques incorporating constraints at each iteration [1].

The benefits of using STEM HAADF images for subsequent tomographic reconstruction of nanoscale objects are shown in Figure 2. This shows a heterogeneous catalyst, composed of $\mathrm{Ru}_{10} \mathrm{Pt}_{2}$ nanoaprticles supported within mesoporous silica (MCM-41), developed for the hydrogenation of trans-trans muconic acid (derived from sugar) and its conversion into adipic acid, a necessary precursor for the production of nylon [5]. What is clear from this comparison is that, whilst the mesopores are seen in the bright-field image, the nanoparticles are invisible. In the STEM image both the nanoparticles and the pore walls are evident.

Figure 3 shows examples of tomographic reconstructions made using STEM HAADF images, all acquired using a FEI Tecnai F20 microscope and a Fischione ultra high tilt holder. Figure 3(a) illustrates how, for a catalyst system, both the distribution of nanoparticles (arrowed) and the surface morphology of the supporting medium, silica in this case, can be visualised from a tomographic reconstruction. Figure 3(b) shows a multiwalled carbon nanotube in which iron catalyst has been found embedded within the nanotube's core. The serpentine nature of this nanotube is evident from the reconstruction. Figure 3(c) shows a slice from a reconstruction of another multiwall nanotube in which the fine filamentary nature of the internal 'bridges' has been revealed with remarkable clarity. In order to increase the signal to noise of the image the inner angle of the HAADF detector was reduced from a typical $40 \mathrm{mrad}$ to only $20 \mathrm{mrad}$. However in this case this did not lead to any gross artefacts or unwanted diffraction contrast. 
References

[1] P.A.Midgley and M.Weyland, Ultramicroscopy 96 (2003)413.

[2] P.A.Midgley, et al., Chem. Comm. 18 (2001) 907.

[3] G. Mobus and B.J. Inkson, Applied Physics Letters 79 (9) (2001) 1369.

[4] M. Weyland and P.A. Midgley, Microsc. Microanal 9 (2003) 542.

[5] J.M. Thomas et al., Accounts of Chemical Research 36 (2003) 20.

[6] We thank the EPSRC, Isaac Newton Trust, FEI Company and the Royal Society for financial support.

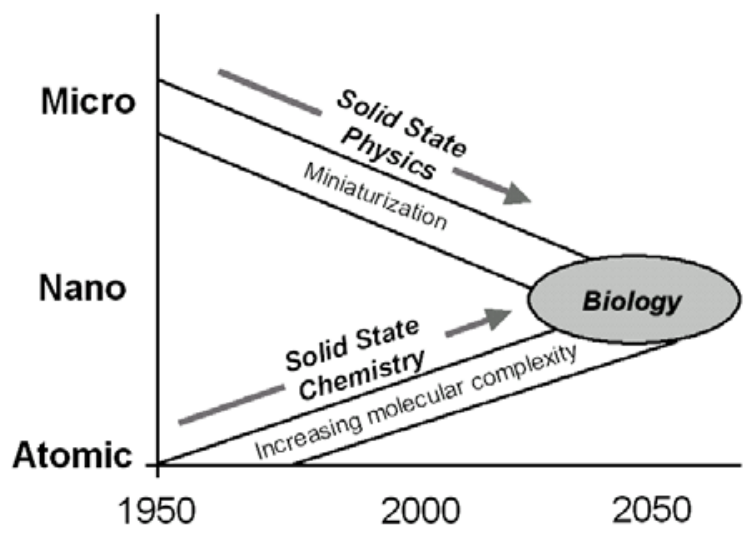

Fig. 1. An illustration as to how, in nanoscale materials science of the future, there may be a need to analyse $3 \mathrm{D}$ devices as complex as biological structures.

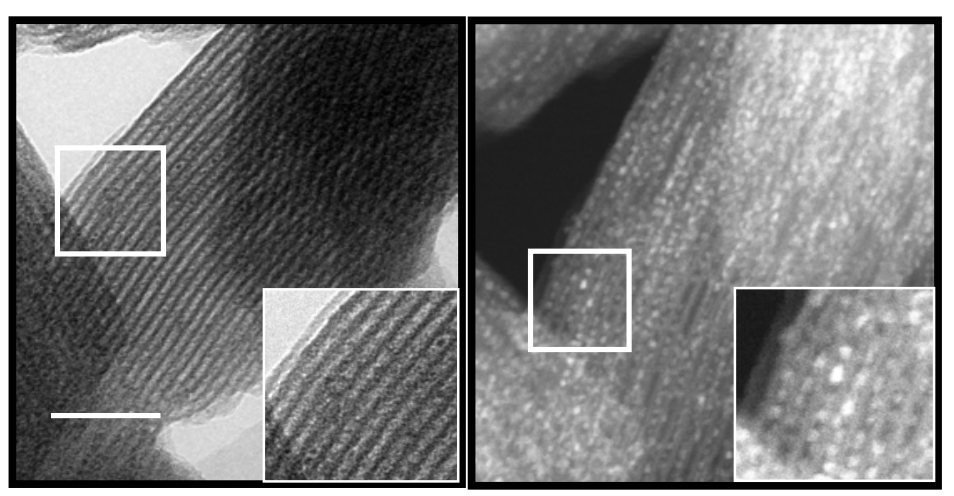

Fig. 2. A comparison of the same region of a heterogeneous catalyst (Pd-Ru in MCM41) using bright-field TEM and dark-field STEM images. Only the pores are shown in the TEM image, whereas both pore walls and particles are evident in the STEM image.
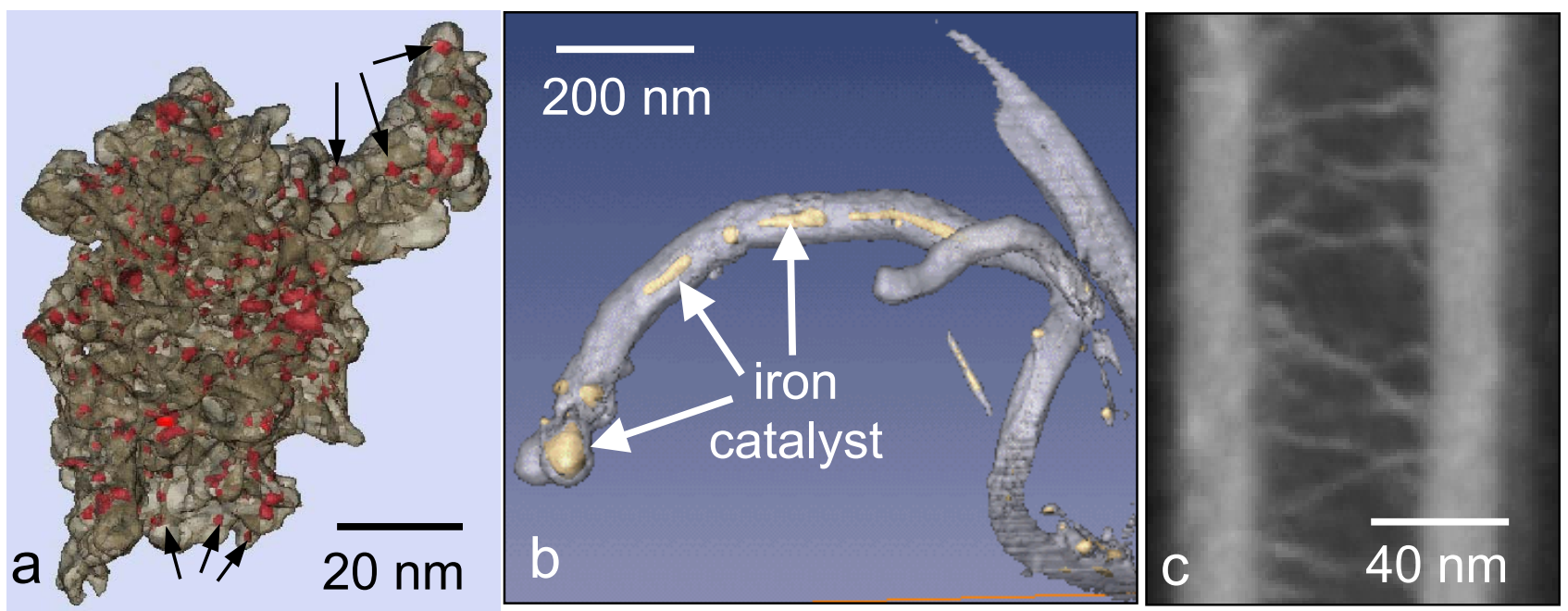

Fig. 3. (a) A three-dimensional reconstruction (surface render) of a catalyst composed of Ru-rich nanoparticles embedded within a highly porous silica support. Examples of the nanoparticles are arrowed. (b) A surface render of a multi-wall nanotube showing iron particles embedded within the tube core. (c) A slice through a reconstruction of a multi-wall nanotube showing fine filamentary bridging structures of only $1-2 \mathrm{~nm}$ in width. 